\title{
Comportamiento y decisión cardiovascular en el síndrome de MASS. Reporte de un caso y revisión de la literatura
}

\section{Cardiovascular behavior and decision in MASS syndrome. A case report and literature review}

\section{Mauricio D. Gómez-González ${ }^{1 *}$, Mariana Tapia-Paredes², Luis de Jesús Colín-Lizalde Francisco González-Ruíz $z^{4}$, Valentín Herrera-Alarcón ${ }^{1}$ y María E. Soto-López ${ }^{5}$}

${ }^{1}$ Servicio de Cirugía Cardiovascular, Centro Médico ABC; ${ }^{2}$ Departamento de servicio social, Universidad La Salle; ${ }^{3}$ Servicio de Electrofisiología, Centro Médico ABC; ${ }^{4}$ Servicio de Terapia intensiva posquirúrgica y Ecocardiografía, Instituto Nacional de Cardiología Ignacio Chávez; ${ }^{5}$ Servicio de Investigación, Centro Médico ABC. Ciudad de México, México

\begin{abstract}
Resumen
El síndrome de MASS (que afecta a: válvula mitral [M], aorta [A], piel [S, skin], sistema musculoesquelético [S, skeletal]) es una rara enfermedad genética con un fenotipo similar al del síndrome de Marfan, pero con diferencias cardiovasculares importantes, como ausencia de afección de la raíz aórtica y marcada afectación mitral. Presentamos el caso de una paciente con síndrome de MASS y revisamos la limitada literatura con respecto a estas diferencias; finalmente discutimos acerca del impacto que pudiera tener esta información con respecto a las decisiones desde el punto de vista de la cirugía cardiovascular.
\end{abstract}

Palabras clave: Síndrome de MASS. Síndrome de Marfan. Síndrome de prolapso valvular mitral. Prolapso valvular mitral. Espectro Marfan.

\begin{abstract}
MASS syndrome (disorder of connective tissue characterized by involvement of the mitral valve, aorta, skeleton, and skin) is a rare genetic disease with a phenotype similar to that of Marfan syndrome, but with important cardiovascular differences like the absence of aortic root aneurysm and marked mitral affection. We present a case of a patient with MASS syndrome and review the limited literature addressing these differences, and we discuss the impact this information may have in decisions regarding cardiovascular surgery.
\end{abstract}

Key words: MASS syndrome. Marfan syndrome. Mitral valve prolapse syndrome. Mitral valve prolapse. Marfan spectrum.

Correspondencia:

*Mauricio D. Gómez-González

E-mail: mauricio_damian@surgical.net

DOI: 10.24875/ACM.20000397
Disponible en internet: 06-09-2021 Arch Cardiol Mex. 2021;91(4):480-484

www.archivoscardiologia.com 1405-9940 / @ 2020 Instituto Nacional de Cardiología Ignacio Chávez. Publicado por Permanyer. Este es un artículo open access bajo la licencia CC BY-NC-ND (http://creativecommons.org/licenses/by-nc-nd/4.0/). 


\section{Introducción}

En 1989 Glesby y Pyeritz crearon el acrónimo MASS para describir el fenotipo de válvula mitral (prolapso), aorta (dilatación leve), esqueleto (skeleton) y piel (skin), que observaron en algunos pacientes con diagnóstico de síndrome de Marfan, pero que no cumplían con todas las características y parecían tener un curso más leve de la enfermedad ${ }^{1}$. En 1996 los criterios de Ghent-1 para el diagnóstico de síndrome de Marfan confirmaron la presencia de pacientes tipo Marfan con marcada afección mitral, afección aórtica leve, involucro esquelético y estrías cutáneas; sin embargo, no se profundizó en los criterios diagnósticos para el síndrome de MASS y estos quedaron poco claros².

Los criterios revisados de Ghent-2 (2010) ahondaron en la presencia de otros síndromes parecidos al Marfan y refinaron los criterios diagnósticos para estos y su diferenciación. Se estableció que el síndrome de MASS es un síndrome tipo Marfan con prolapso valvular mitral (PVM), sin aneurisma de la raíz aórtica (z-score $\geq 2$ ), sin ectopia lentis y que además requiere un score sistémico de Ghent mayor a 5 con al menos una afección esquelética. Cuando el paciente tiene aspecto leptosómico, PVM, ausencia de aneurisma en la raíz aórtica y ausencia de ectopia lentis, pero no alcanza 5 puntos en el score sistémico de Ghent, queda catalogado como síndrome de prolapso valvular mitral (SPVM) aislado ${ }^{3}$. En varios de estos estudios se comentó que si bien en el MASS los pacientes no tienen aneurisma aórtico, sí cursaban con dilatación aórtica limítrofe ${ }^{1,4,5}$.

\section{Presentación del caso}

Se trata de una paciente de 23 años que inició un año antes con síndrome de muerte súbita abortada y que posterior al evento recibió un desfibrilador automático implantable (DAI), el cual desfibriló dos ocasiones más de manera ambulatoria. A la exploración física, con estatura de $1.75 \mathrm{~m}$, longilínea, facies alargada, brazos y piernas largas, aracnodactilia y estrías violáceas en abdomen, cadera y piernas. Historia de cirugía de columna para corrección de xifoescoliosis toracolumbar a los 8 años de edad y sin antecedente de problemas oftalmológicos. El aspecto marfanoide en general era leve y el score sistémico de Ghent de 7 . Los padres no tenían habitus leptosómico.

Ecocardiográficamente se encontró prolapso mitral clásico con insuficiencia valvular grave con efecto Coanda, redundancia de ambas valvas con predominio de la anterior, dilatación importante del atrio izquierda $\left(64 \mathrm{ml} / \mathrm{m}^{2}\right)$, ausencia de otras valvulopatías y anillo tricuspídeo no dilatado, dilatación ventricular izquierda con diámetro diastólico de $63 \mathrm{~mm}$, fracción de eyección del ventrículo izquierdo (FEVI) del 46\%, masa ventricular izquierda indexada de $161 \mathrm{~g} / \mathrm{m}^{2}$, función ventricular derecha adecuada y presión sistólica de la arteria pulmonar de $36 \mathrm{mmHg}$. Tomográficamente se encontró una aorta torácica con diámetro anular de $21 \mathrm{~mm}$, segmento sinusal de $34 \mathrm{~mm}$, unión sinotubular $27 \mathrm{~mm}$, aorta ascendente $32 \mathrm{~mm}$ y cayado aórtico de $27 \mathrm{~mm}$; sin lesiones coronarias por este método. Al interrogatorio del DAI se encontró ritmo sinusal de base, con abundantes episodios de taquicardia ventricular no sostenida y dos episodios de fibrilación ventricular adecuadamente abortados por el dispositivo. Se interconsultó a un especialista en enfermedades del tejido conectivo, quien diagnosticó síndrome de MASS.

Se sometió a cambio valvular mitral por prótesis mecánica St. Jude Reagent $27 \mathrm{~mm}$ con reducción circunferencial del atrio izquierdo, exclusión de orejuela izquierda y revisión tricuspídea. Ambas valvas mitrales se encontraban aneurismáticas, con redundancia severa y ruptura de algunas cuerdas tendinosas, la insuficiencia mitral era global y el anillo nativo tenía diámetro normal; la válvula tricúspide se encontró sin alteración. El atrio izquierdo era tipo aneurismático, con ostium de la orejuela izquierda dilatado y una zona amplia adelgazada en su base. La reducción circunferencial del atrio izquierdo se realizó plicando internamente desde el techo de la cavidad, dirigido hacia el ostium de la orejuela y excluyéndolo, llegando finalmente casi hasta el anillo mitral posterior, todo con un surjete de Carrel reforzado con bandas de Teflón®. Hubo un fracaso al destete de bomba, de la cual salió en el segundo intento. Tiempo de circulación extracorpórea: 121', tiempo de pinzamiento aórtico: 82'.

Se valoró intraoperatoriamente la factibilidad de una plastia valvular mitral. Dada la mala función ventricular preoperatoria, la necesidad de una reconstrucción valvular compleja y el requerimiento de una plastia extensa del atrio izquierdo, se optó por abreviar el tiempo de circulación extracorpórea con un cambio valvular mitral protésico mecánico. La previa decisión de la paciente de no desear tener descendencia facilitó esta decisión.

El postoperatorio transcurrió sin eventualidades, requirió apoyo vasopresor a dosis bajas el primer día y apoyo inotrópico con levosimendán por 48 horas; egresó a domicilio seis noches después. Durante los primeros meses la paciente mejoró de clase funcional III a I; el lastre arritmógeno disminuyó en interrogatorios subsecuentes al DAl y no tuvo descargas nuevas. El 
control ecocardiográfico al año mostró normalización de la FEVI y la presión pulmonar.

\section{Comportamiento cardiovascular del síndrome de MASS}

Al igual que en el síndrome de Marfan, se supone que la etiología del MASS son mutaciones en el gen de la fibrilina 1 (FBN1), pero se sospecha que el carácter relativamente limitado del síndrome es producto de mutaciones pequeñas en locus menos severos y menos usuales. De acuerdo con la base de datos OMIM (compiladora de síndromes y enfermedades genéticas), el síndrome de MASS es producto de una inserción de cuatro pares de bases (TTCA) en el nucleótido 5138 del gen de la FBN1 (FBN1, 4-BP INS, NT5138); sin embargo, dicha tipificación es antigua (1993) y no ha sido corroborada de manera sistemática ${ }^{6}$. Actualmente el síndrome de MASS se diagnostica de manera clínica y por exclusión, pues no existe prueba genética validada para su uso sistemático ${ }^{3}$. Los pacientes diagnosticados como MASS que no tienen mutaciones típicas de Marfan, por su evolución clínica, prácticamente siempre permanecen clasificados como MASS; en cambio, los pacientes diagnosticados como MASS que tienen mutaciones típicas de Marfan pudieran terminar quedando catalogados como Marfan de acuerdo con su evolución clínica ${ }^{7}$. No existe una incidencia reporta$\mathrm{da}$, pero en diferentes estudios ${ }^{5,8}$ entre el 0.5 y $2.4 \%$ de los pacientes inicialmente sospechosos de Marfan terminaron quedando diagnosticados como MASS.

El comportamiento clínico del síndrome MASS y del SPVM fue comparado con el de Marfan por Rippe y de Backer en 2016, cuando, acotándose por los criterios de Guent-2, analizaron de manera longitudinal retrospectiva a 44 adultos diagnosticados como MASS o SPVM, frente a 81 pacientes con Marfan (seguimiento de $66 \pm 49$ meses); también se les hizo análisis de los genes FBN1, TGFBR1 y TGFBR2 a todos.

Las diferencias basales cardiovasculares y algunas no cardiovasculares más importantes que encontraron fueron las siguientes ${ }^{7}$ :

- El z-score de la porción aórtica sinusal fue menor a 2 para todos los pacientes con MASS y SPVM, mientras que la mayoría de los pacientes con Marfan tenían mediciones superiores a $2(p<0.001)$. Con el cálculo del z-score por el método de Deveraux, algunos pacientes con MASS tuvieron mediciones limítrofes altas o levemente superiores a 2, mientras que ninguno de SPVM las tuvo.
- La prevalencia de prolapso mitral anterior fue similar para los tres síndromes, mientras que el prolapso mitral posterior y bivalvar fue más frecuente en el Marfan. Sin embargo, la prevalencia de insuficiencia mitral moderada a severa fue alrededor de dos veces más frecuente en el MASS y el SPVM que en el Marfan (27, 38 y 16\%, respectivamente, $p=0.049$ ).

- Los pacientes con Marfan tuvieron scores sistémicos de Ghent mayores a los de MASS, y los de MASS tuvieron scores mayores a los del SPVM (todos $p<0.001$ ).

- Ningún paciente con MASS o SPVM tuvo mutaciones en los genes analizados; los que tuvieron mutaciones específicas del Marfan fueron excluidos independientemente del diagnóstico clínico. Todos los pacientes con Marfan tuvieron mutaciones conocidas del gen FBN1 causantes de Marfan.

En el seguimiento retrospectivo los hallazgos cardiovasculares más importantes fueron?:

- A los 47 años de edad, la mitad de los pacientes con síndrome de Marfan ya habían recibido cirugía de aorta, mientras que ningún paciente con MASS o SPVM la requirió (Figura 1). Además, cuatro pacientes con Marfan murieron ambulatoriamente, siendo una causa aórtica lo más probable; un paciente con SPVM murió de causa desconocida, mientras que ningún paciente con MASS murió.

- A los 69 años de edad, el 56\% de los pacientes con MASS/SPVM ya habían recibido cirugía mitral; mientras que a los 58 años, el $58 \%$ de los pacientes con Marfan también habían recibido ya cirugía mitral. O sea que el riesgo acumulado de recibir cirugía mitral en pacientes con MASS o SPVM vs. Marfan es muy similar, pero con las siguientes diferencias: los pacientes con MASS/ SPVM recibieron cirugía mitral como su primera y única intervención cardiovascular a una edad más joven, mientras que los pacientes con Marfan sometidos a cirugía mitral la recibieron a una mayor edad y frecuentemente como una segunda intervención cardiovascular (siendo la primera una cirugía aórtica) (Figura 2).

\section{Decisión cardiovascular en el síndrome de MASS}

El cardiólogo y el cirujano cardiovascular deben estar familiarizados con la existencia del MASS y deben ser capaces de sospecharlo en ciertos pacientes; el diagnóstico es clínico y complejo, debiendo limitarse a especialistas en enfermedades del tejido conectivo. No existe prueba genética para diagnosticar MASS, pero la ausencia de mutaciones específicas de Marfan refuerza importantemente el diagnóstico. 

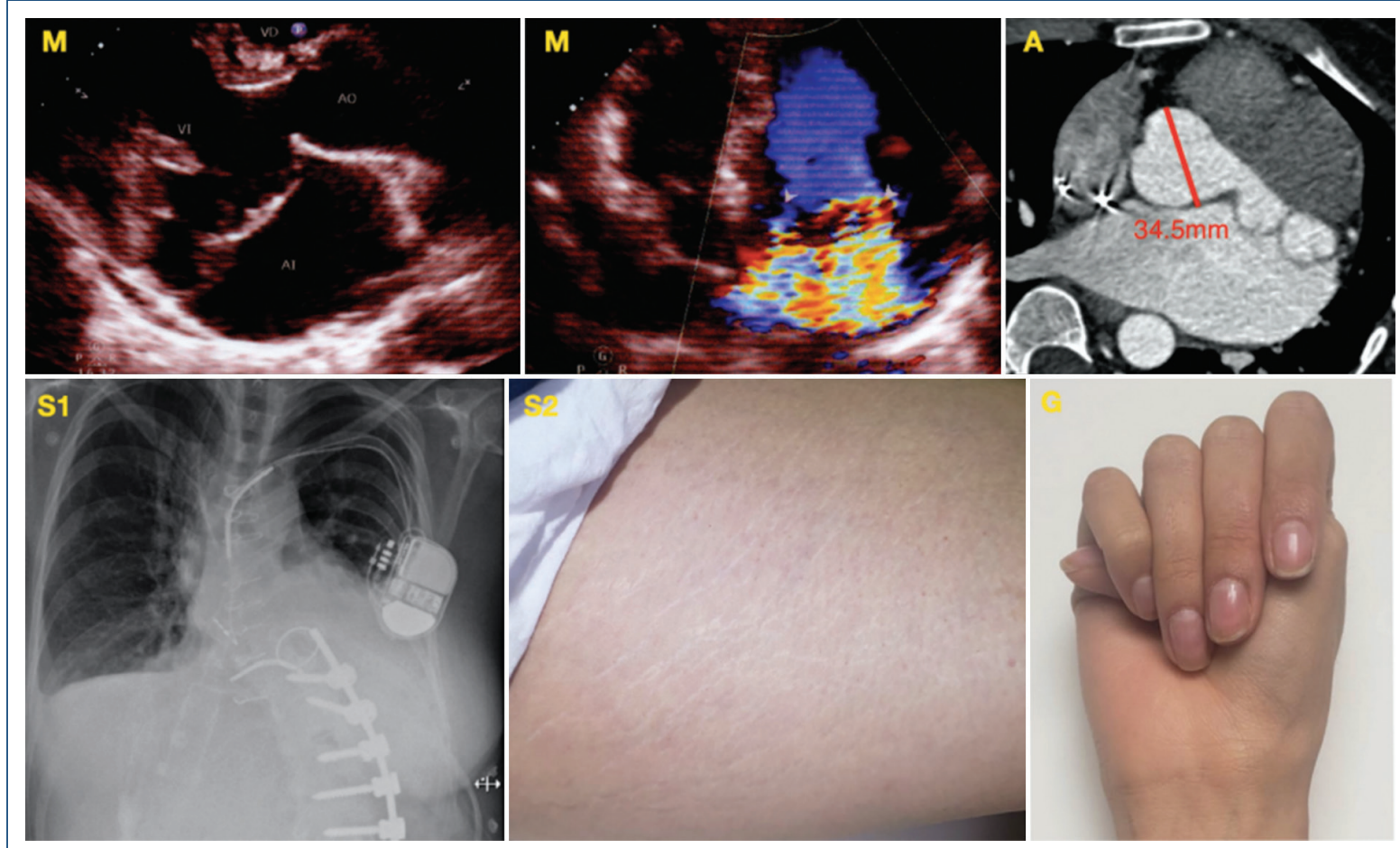

Figura 1. Acrónimo de MASS en la paciente del caso. M (mitral): prolapso mitral con insuficiencia severa. A (aorta): ausencia de aneurisma de la raíz aórtica (se observa también prolapso mitral y dilatación importante del atrio izquierdo). $S 1$ (skeleton): alteraciones esqueléticas representadas por cifoescoliosis toracolumbar y antecedente de cirugía para esta. S2 (skin): estrías cutáneas. G (Ghent): signo del pulgar positivo, el cual forma parte del score sistémico de Ghent.

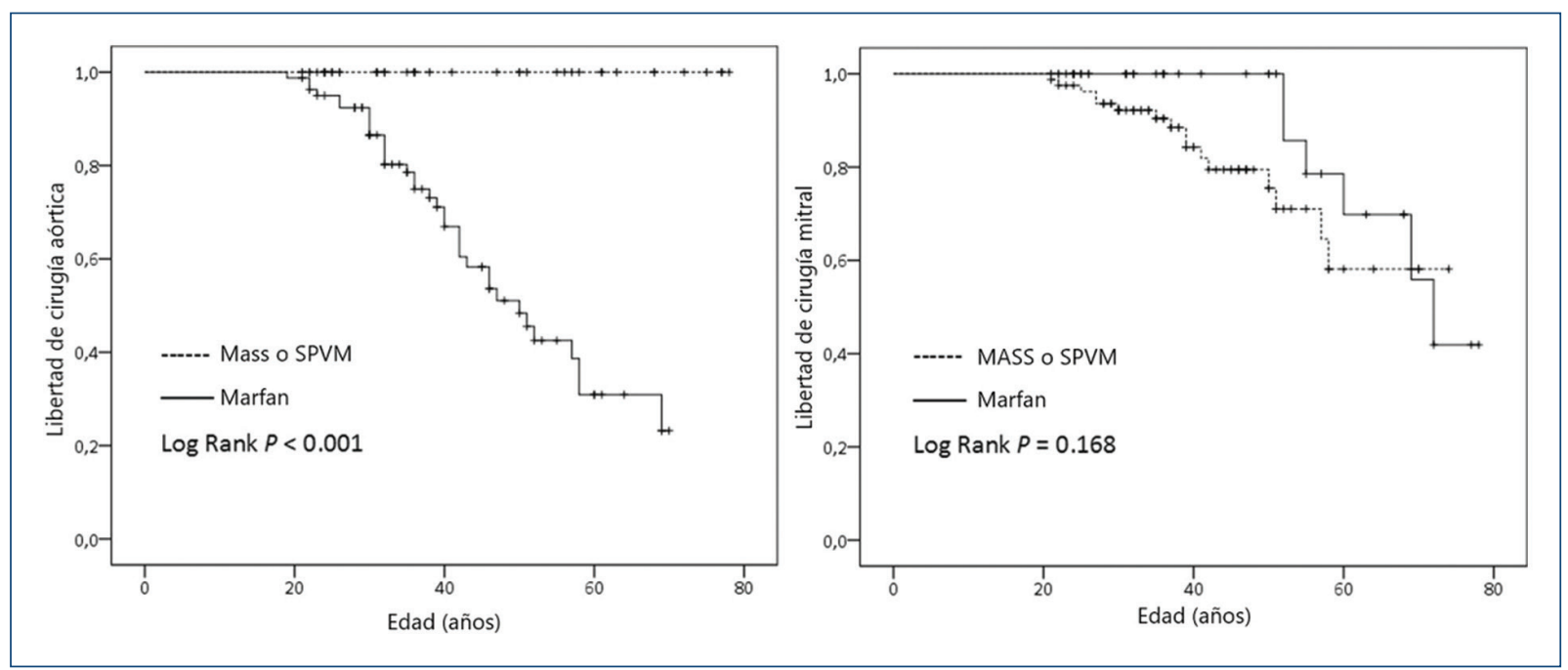

Figura 2. Curvas de Kaplan-Meier comparando la libertad de cirugía aórtica y cirugía mitral en los tres síndromes (adaptada de Rippe, et al., 2016').

El estudio de Rippe y de Becker es el único que ha descrito el comportamiento en el tiempo del MASS y el SPVM, pero su principal limitante es el carácter retrospectivo y el tiempo de seguimiento; hasta que se conozca el comportamiento clínico a muy largo plazo, parece sensato dar seguimiento a ambos síndromes. 
Tabla 1. Características positivas del score sistémico de Ghent de la paciente del caso

\begin{tabular}{|l|c|}
\hline Característica & Valor \\
\hline Signo del pulgar y signo de la muñeca & +3 puntos \\
\hline Escoliosis o cifosis toracolumbar & +1 \\
\hline 3 de 5 características faciales & +1 \\
\hline Estrías de la piel & +1 \\
\hline Prolapso de la válvula mitral & +1 \\
\hline Total & 7 puntos \\
\hline
\end{tabular}

Tabla 2. Criterios diagnósticos para el síndrome de MASS y el de prolapso valvular mitral ${ }^{3}$

\begin{tabular}{|l|l|}
\hline Síndrome de MASS & Síndrome de prolapso valvular mitral \\
\hline $\begin{array}{l}\text { Presencia de } \\
\text { prolapso valvular } \\
\text { mitral }\end{array}$ & Presencia de prolapso valvular mitral \\
\hline $\begin{array}{l}\text { Ausencia de } \\
\text { aneurisma de la raíz } \\
\text { aórtica(z-score<2) }\end{array}$ & $\begin{array}{l}\text { Ausencia de aneurisma de la raíz } \\
\text { aórtica(z-score<2) }\end{array}$ \\
\hline $\begin{array}{l}\text { Ausencia de ectopia } \\
\text { lentis }\end{array}$ & Ausencia de ectopia lentis \\
\hline $\begin{array}{l}\text { Aspecto leptosómico } \\
\text { con score sistémico } \\
\text { de Ghent }>5 \text { (con al } \\
\text { menos una condición } \\
\text { esquelética) }\end{array}$ & $\begin{array}{l}\text { Aspecto leptosómico con score } \\
\text { sistémico de Ghent }<5\end{array}$ \\
\hline
\end{tabular}

MASS: válvula mitral (M), aorta (A), piel (S, skin), sistema musculoesquelético (S, skeletal).

En particular, los pacientes con diagnóstico de MASS sin análisis genético del gen FBN1 deben ser seguidos sistemáticamente para descartar que se trate de un Marfan incipiente y evitar el retraso en la detección de condiciones aórticas.

Los pacientes con MASS/SPVM muy probablemente no requieran procedimientos aórticos; el umbral de indicación debe ser muy conservador, incluso en pacientes con dilatación limítrofe. La cirugía mitral en el MASS/ SPVM debe ser precoz a fin de disminuir el daño cardiaco, ya que los parámetros de severidad hemodinámica y repercusión ventricular secundaria a insuficiencia mitral que son indicación de cirugía se alcanzan a edades más jóvenes que en insuficiencias mitrales de otras causas, incluyendo el Marfan. La decisión entre reemplazo vs. plastia mitral no ha sido analizada en el contexto de estos síndromes, pero se reportan ambos procedimientos; existe una preocupación teórica de realizar una plastia sobre valvas mitrales que están estructuralmente alteradas por una causa genética. El adecuado diagnóstico permitirá ajustar de manera óptima el momento y la envergadura de las cirugías cardiovasculares en el MASS, principalmente evitando intervenciones aórticas innecesarias, así como intervenciones mitrales tardías.

\section{Financiamiento}

No se recibió ningún financiamiento.

\section{Conflictos de intereses}

Los autores declaran no tener conflicto de intereses.

\section{Responsabilidades éticas}

Protección de personas y animales. Los autores declaran que para esta investigación no se han realizado experimentos en seres humanos ni en animales.

Confidencialidad de los datos. Los autores declaran que han seguido los protocolos de su centro de trabajo sobre la publicación de datos de pacientes.

Derecho a la privacidad y consentimiento informado. Los autores han obtenido el consentimiento informado de los pacientes y/o sujetos referidos en el artículo. Este documento obra en poder del autor de correspondencia.

\section{Bibliografía}

1. Glesby MJ, Pyeritz RE. Association of mitral valve prolapse and systemic abnormalities of connective tissue, a phenotypic continuum. JAMA. 1989;262(4):523-8.

2. De Paepe A, Devereux RB, Dietz HC, Hennekam RC, Pyeritz RE. Revised diagnostic criteria for the Marfan syndrome. Am $\mathrm{J}$ Med. 1996;62(4):417-26.

3. Loeys BL, Dietz HC, Braverman AC, Callewaert BL, de Backer J, Devereux $\mathrm{RB}$, et al. The revised Ghent nosology for the Marfan syndrome. J Med Genet. 2010;47(7):476-85

4. Pyeritz RE. Evaluation of the adolescent or adult with some features of Marfan syndrome. Genet Med. 2012;14(1):171-7.

5. Radonic T, de Witte P, Groenink M, de Bruin-Bon RACM, Timmermans $\mathrm{J}$, Scholte AJH, et al. Critical appraisal of the revised Ghent criteria for diagnosis of Marfan syndrome. Clin Genet. 2011;80:346-53.

6. Dietz HC, McIntosh I, Sakai LY, Corson GM, Chalberg SC, Pyeritz RE et al. Four novel FBN1 mutations: significance for mutant transcript level and EGF-like domain calcium binding in the pathogenesis of Marfan syndrome. Genomics. 1993;17:468-75.

7. Rippe M, de Backer J, Kutsche K, Mosquera LM, Schüler H, von Kodolitsch Y. Mitral valve prolapse syndrome and MASS phenotype: Stability of aortic dilatation but progression of mitral valve prolapse. Int $\mathrm{J}$ Cardiol Heart Vasc. 2016:10:39-46.

8. Gentilini D, Oliveria A, Fazia T, Pini A, Marelli S, Bernardinelli L, et al. NGS analysis in Marfan syndrome spectrum: Combination of rare and common genetic variants to improve genotype-phenotype correlation analysis. PLoS One. 2019;14(9):e0222506. 